Reprod. Nutr. Dévelop., 1988, 28 Suppl. n¹, 187-188

\title{
Utilisation de l'eau lourde pour l'estimation de la composition corporelle de béliers peul-peul au Sénégal
}

\section{RICHARD}

avec la collaboration de B. AHOKPE et A. KA

IEMVT-CIRAD et

ISRA, Laboratoire national de l'Elevage et de Recherches vétérinaires, B.P. 2057 - Dakar, Sénégal.

Summary. Prediction of total body water and body fat was studied using deuteriated water $\left(\mathrm{D}_{2} \mathrm{O}\right)$ in $14 \mathrm{Peul}$ peul rams. Body fat content ranged between 1.95 and $16.7 \%$ of the empty body weight. The $\mathrm{D}_{2} \mathrm{O}$ diffusion space gave an accurate prediction when body weight was included in the equation.

La connaissance de l'évolution de la composition corporelle au cours de la croissance et de l'engraissement est une étape nécessaire à l'évaluation des besoins des animaux. Elle est quasiment inexistante en Afrique tropicale. Dans le but d'estimer les besoins énergétiques et azotés des ovins au Sénégal, des études de la composition corporelle sont en cours dans le cadre d'un programme de recherche franco-sénégalais associant I'IEMVT-CIRAD et le LNERV-ISRA. Ces études s'accompagnent de mesures indirectes de l'eau corporelle à partir de l'espace de diffusion de l'eau lourde. Ces dernières sont rapportées ci-dessous.

Matériel et méthodes. L'étude a porté sur 14 béliers entiers de race peul-peul âgés de 10 à 20 mois, alimentés avec de la paille de riz ou des fanes d'arachide et un concentré pour certains d'entre eux.

Les animaux devant recevoir l'injection d'eau lourde ont été tirés au hasard parmi l'ensemble des lots expérimentaux en début et en fin d'essai. Les animaux abattus ont fait l'objet d'une discussion complète. Le sang, les muscles, le cinquième quartier, les os, le cuir ont été séparés. Les teneurs en matière sèche ont été mesurées à l'étuve à $105^{\circ} \mathrm{C}$ et en lyophilisateur. Les échantillons lyophilisés ont été broyés puis analysés pour les cendres et l'azote.

Les injections d'eau lourde ont été faites selon la technique décrite par Robelin (1977), à raison de $0,6 \mathrm{~g}$ de $\mathrm{D} 20$ par $\mathrm{kg}$ de poids vif. Les prélèvements de sang ont été effectués à $6,8,24$ et $30 \mathrm{~h}$ après l'injection, les moutons étant abattus dès le dernier prélèvement fait. Les animaux ont été pesés au moment de l'injection (PVI), $6 \mathrm{~h}$ après (PV6), puis $30 \mathrm{~h}$ après, ce qui correspond au poids vif à l'abattage (PVA) (tabl. 1).

L'extraction de l'eau lourde et son dosage ont été faits par lyophilisation et spectrophotométrie infrarouge selon les méthodes décrites par Tissier et al. (1978). Cette phase de l'étude a été effectuée par le laboratoire de production ovine du CRZV de Theix que nous tenons à remercier. 
Résultats et discussion. Les teneurs en lipides des animaux disséqués varient dans des limites importantes, les lipides représentant entre 1,95 et $16,7 \%$ du poids vif vide (tabl. 1). II existe une relation étroite entre la quantité de lipides $(L)$ et le poids vif vide et la teneur en eau du corps vide $(R 2=0,995)$.

TABL. 1. - Poids vif à l'abattage et composition chimique des béliers.

\begin{tabular}{lccr}
\hline & Moyenne & Ecart-type & Extrêmes \\
\hline Poids vif à l'abattage $(\mathrm{kg})$ & 26,62 & 4,84 & $19,2-34,6$ \\
Poids vif vide $(\mathrm{kg})$ & 20,07 & 5,79 & $12,9-29,9$ \\
Eau du corps entier $(\mathrm{kg})$ & 18,89 & 2,36 & $14,5-22,7$ \\
Eau du corps vide $(\mathrm{kg})$ & 12,87 & 3,33 & $9,2-18,6$ \\
Lipides $(\mathrm{kg})$ & 2,08 & 1,46 & $0,26-4,99$ \\
Protéines $(\mathrm{kg})$ & 3,57 & 1,14 & $2,34-5,39$ \\
Cendres $(\mathrm{kg})$ & 0,94 & 0,21 & $0,60-1,29$ \\
\hline
\end{tabular}

L'espace de diffusion de l'eau lourde (ECD20) permet d'obtenir une bonne prévision de la quantité d'eau corporelle totale (ECT). L'équation la plus précise est obtenue en ajoutant la différence entre le poids $6 \mathrm{~h}$ après l'injection et celui à l'abattage :

$\mathrm{ECT}(\mathrm{kg})=3,886+0,813 \mathrm{ECD} 20-1,133(\mathrm{PV} 6-\mathrm{PVA}) \quad \mathrm{ETR}=0,585 \quad \mathrm{R} 2$ $=0,948 \mathrm{n}=14$.

Cet ETR représente $3,1 \%$ de l'eau corporelle totale mesurée lors des̀ dissections, pourcentage voisin de ceux rapportés par Robelin (1977) pour des agneaux et par Tissier et al. (1983) pour des brebis en France.

La quantité de lipides $(L)$ peut être estimée à partir de l'espace de diffusion de l'eau lourde. La précision obtenue avec D20 est supérieure (équation 1) à celle obtenue à partir du poids vif des animaux vivants $30 \mathrm{~h}$ après l'injection (PVA) (équation 2) et du poids vif vide (PVV) des animaux abattus (équation 3 ) :

$\mathrm{L}(\mathrm{kg})=-2,735+0,450 \mathrm{PVI}-0,362 \mathrm{ECD} 20 \mathrm{ETR}=0,290 \quad \mathrm{R} 2=0,966$ (1)

$\mathrm{L}(\mathrm{kg})=-5,346+0,279$ PVA $\quad \mathrm{ETR}=0,577 \quad \mathrm{R} 2=0,855$

$L(k g)=-2,841+0,245$ PVV

$\mathrm{ETR}=0,356 \quad \mathrm{R} 2=0,944$

Le coefficient de variation résiduelle est de 13,9\%. Il est légèrement supérieur à celui rapporté par Robelin (1977) pour des agneaux sevrés $(10,5 \%)$. Ce coefficient de variation peut être réduit si les animaux sont abattus et les matières sèches mesurées pour les éléments disséqués : il est alors de 6,2\% par rapport au PVI. Toutefois, la précision avec l'eau lourde reste bonne dans l'ensemble des mesures faites lors des abattages. Elle permet d'envisager le calcul des lipides totaux sur les mêmes animaux en début et en fín d'expérience et de faire des suivis de composition corporelle dans le milieu traditionnel d'élevage.

Robelin J., 1977. Ann. Biol. anim. Bioch. Biophys., 17, 95-105.

Tissier M., Robelin J., Purroy A., Geay Y., 1978. Ann. Biol. anim. Bioch. Biophys., 18, 1223-1228.

Tissier M., Thériez M., Purroy A., Bocquier F., 1983. Reprod. Nutr. Dévelop., 23, 693-707. 